\title{
Actinomicosis diseminada con compromiso de sistema nervioso central
}

\author{
Juan Vásquez, Carlos Gómez, Angela Chiquillo y Luz Pescador
}

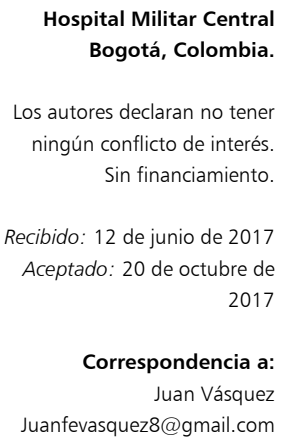

Correspondencia a: Juan Vásquez Juanfevasquez8@gmail.com

\section{Disseminated actinomycosis with central nervous system involvement}

Actinomycosis is very rare, as well as the central nervous system (CNS) condition associated with it, presenting a mortality up to $28 \%$. Its manifestations could be similar to infectious conditions from other etiologies, thus, having a better understanding of the entity increases clinical suspicion and also it can provide a timely treatment. The clinical case of an adult with edema in an upper extremity is presented as a manifestation of thrombophlebitis and an abscessed axillary lesion, in which actinomycetes infection was confirmed. He presented a haematogenous spread with CNS involvement, with fatal ending.

Key words: Actinomycosis; brain abscess; lymphadenopathy.

Palabras clave: Actinomicosis; absceso cerebral; adenopatías.

\section{Introducción}

L a infección por actinomicetos es una condición poco frecuente. La especie más común es Actinomyces israelli. Su presentación más habitual es el compromiso cérvico-facial, en que se desarrolla un proceso granulomatoso con induración y progresión hasta la abscedación de los tejidos blandos y posterior drenaje, con formación de gránulos de azufre. Presentamos el caso clínico de una actinomicosis diseminada con abscesos cerebrales.

\section{Caso clínico}

Varón de 63 años, de raza mestiza, procedente y residente en la ciudad de Bogotá. Fue admitido al servicio de urgencias por un cuadro de 10 días de evolución de dolor, edema y limitación funcional de la extremidad superior izquierda, sin antecedente de trauma. Además, refería síntomas irritativos urinarios caracterizados por tenesmo, poliaquiuria y orina de mal olor.

Tenía antecedentes de una fibrilación auricular permanente con enfermedad del nodo, en terapia anticoagulante con dabigatrán, hiperplasia prostática e hipotiroidismo. Además, ocho años atrás había sido sometido a una colecistectomía y cursado con una fístula bilio-entérica, la que sólo presentaba cambios cicatriciales.

$\mathrm{Al}$ examen físico de su ingreso se observó una asimetría en las extremidades superiores dada por un edema duro doloroso en la extremidad superior izquierda, además de un conglomerado de adenopatías axilares manifestado como un aumento de volumen axilar indurado doloroso, no móvil de más de $3 \mathrm{~cm}$ de diámetro y un leve tinte ictérico en mucosas.

El edema de la extremidad superior izquierda fue evaluado mediante una ecografía doppler que confirmó una trombosis venosa profunda de la vena braquial. Dentro de los exámenes generales presentaba una leucocitosis de 22.600 cél $/ \mathrm{mm}^{3}$ con neutrofilia (94\%), una colestasis leve (bilirrubina total 2,5 y directa $1,7 \mathrm{mg} / \mathrm{dl}$, GOT $131 \mathrm{U} / \mathrm{L}$, GGT $93 \mathrm{U} / \mathrm{L}$ ), y elevación de reactantes de fase aguda. El examen de orina demostró piuria y bacteriuria, lo que junto con los síntomas irritativos urinarios se interpretó como una infeción urinaria asociada, que se trató con cefuroxima durante siete días.

Frente a una trombosis venosa de presentación y localización inusual, sin factor predisponente aparente, además del antecedente de anticoagulación con un medicamento blanco específico (dabigatrán), se planteó un proceso paraneoplásico versus un estado de trombofilia. Además, se realizó estudio inmunológico con medición de anticuerpos anticardiolipina $\operatorname{IgG}$ e $\operatorname{IgM}$, así como anticuerpos anti $\beta 2$ microglobulina, los cuales fueron negativos.

Se realizó una tomografía (TC) de tórax y abdomen con contraste en que se observó un conglomerado ganglionar axilar izquierdo, con un centro hipodenso sugerente de un proceso inflamatorio necrótico, sin signos de malignidad en relevos ganglionares. En el parénquima pulmonar se observaron áreas de consolidación en los lóbulos superiores de predominio izquierdo, con engrosamiento interlobulillar e imágenes de vidrio esmerilado (Figura 1). En la TC de abdomen se observaron cambios hepáticos post-colecistectomía y escaso líquido libre en la cavidad abdominal. Se planteó realizar una biopsia pulmonar, el que fue rechazado por el paciente. 
Se realizó un drenaje y biopsia quirúrgica del conglomerado ganglionar axilar, obteniéndose $100 \mathrm{ml} \mathrm{de}$ material purulento que se envió para cultivo.

El paciente evolucionó con deterioro del estado neurológico, somnolencia y debilidad en las extremidades, mayor a izquierda y fiebre. Se tomaron hemocultivos que resultaron positivos para bacilos grampositivos.

Se realizó una TC cerebral en que se identificaron lesiones intra-axiales con realce anular en la sustancia blanca periventricular y edema vasogénico periférico (Figura 2) y posteriormente una RM en que se confirmaron lesiones compatibles con abscesos cerebrales. Se realizó un drenaje por esterotaxia, obteniéndose $10 \mathrm{ml}$ de material purulento. En los cultivos de las secreciones ganglionar axilar y cerebral, así como en los hemocultivos hubo desarrollo de Actinomyces odontolyticus.

El paciente había recibido siete días de cefuroxima y 15 días de vancomicina, por la presencia de bacilos grampositivos en los hemocultivos. Con la confirmación microbiológica se ajustó la terapia a ampicilina/sulbactam; sin embargo, el paciente evolucionó con deterioro clínico, progresión a estupor, fibrilación auricular con respuesta ventricular no controlada, edema pulmonar y falla ventilatoria, por lo que se trasladó a una unidad de cuidados intensivos, con pobre respuesta a las medidas instauradas, falleciendo de una falla multiorgánica.

\section{Discusión}

La actinomicosis en una enfermedad infecciosa infrecuente de comportamiento crónico. Es causada por un bacilo grampositivo filamentoso anaeróbico que forma parte de la microbiota de la cavidad oral, tracto gastrointestinal y genitourinario. Se han descrito cerca de 30 especies, todas ellas pertenecientes a la familia Actinobacteria, orden Actinomicetales y se relacionan con otros géneros como Corynebacterium, Mycobacterium, Nocardia y Propionibacterium ${ }^{1}$. Inicialmente el género Actinomyces se consideró un hongo por su aspecto filamentoso y el aparente desarrollo de micelios, pero la falta de glucanos y de quininas en las paredes externa y nuclear, así como la ausencia de ribosomas hizo que se reclasificara como bacteria ${ }^{2}$.

$\mathrm{Su}$ incidencia es poco conocida, probablemente por un subdiagnóstico dado por su presentación semejante con otras condiciones, como neoplasias o infecciones por micobacterias ${ }^{3}$. Algunas publicaciones realizan una aproximación de la incidencia entre 0,3-1 episodio por cada 100.000 habitantes/año ${ }^{2}$.

El cuadro clínico se presenta como un proceso endógeno que puede acompañarse de aislamientos de especies "comensales", que de alguna manera ayudan a Actinomyces a sobrevivir".
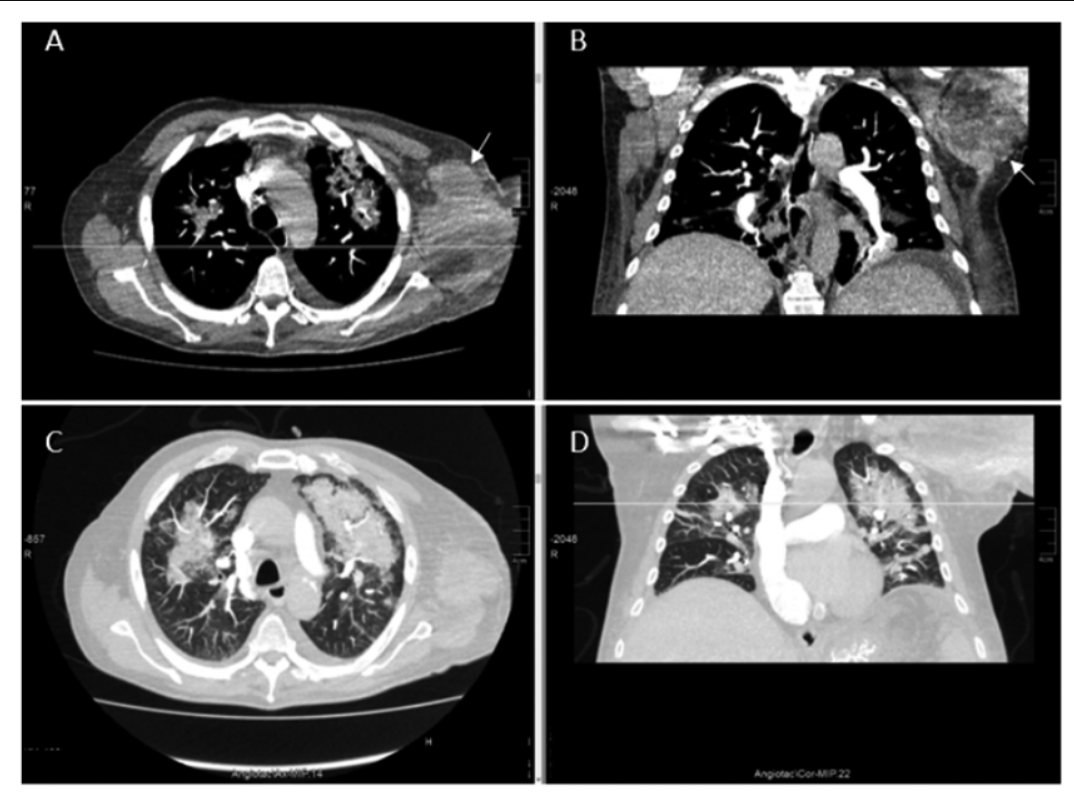

Figura 1. A, B: Lesión axilar izquierda hipodensa correspondiente a una colección de contenido heterogéneo (flechas blancas). B, C: Consolidación de un patrón broncocéntrico, compromiso multilobar con respeto parcial de la periferia, derrame pleural bilateral.

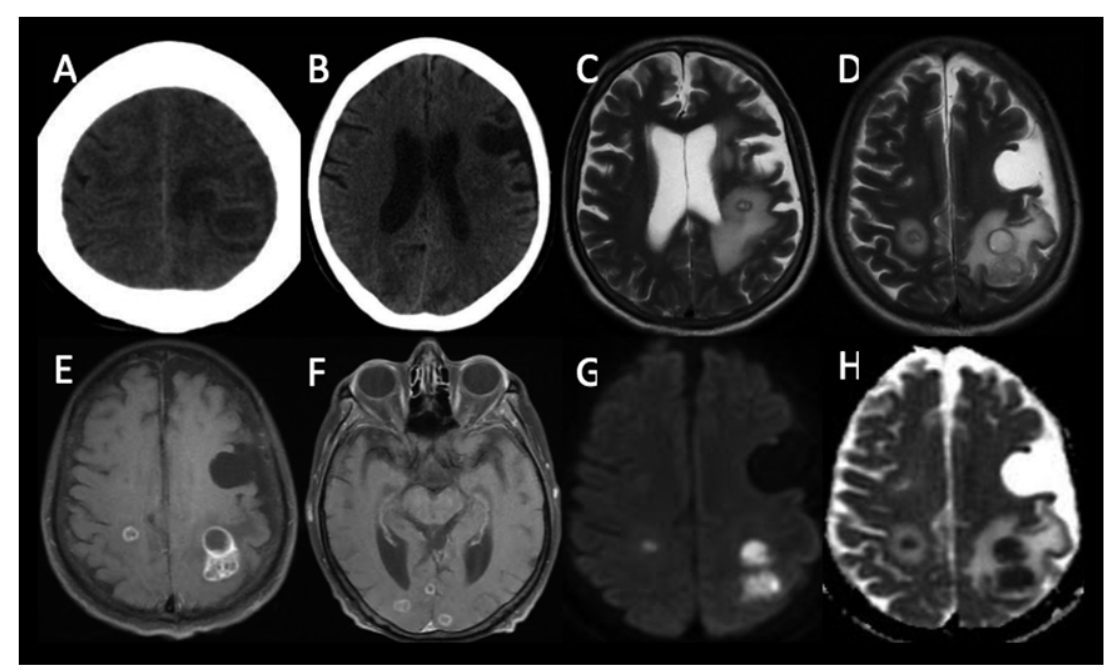

Figura 2. A, B: TC axial simple muestra colecciones hipodensas de contornos bien definidos y paredes hiperdensas regulares en región frontotemporal y corona radiada izquierda acompañadas de edema vasogénico adyacente. C, D: RM T2 demuestra las múltiples colecciones hiperintensas con halo hipointenso e importante edema vasogénico adyacente, en ambos hemisferios cerebrales. E, F: RM T1 + MC Colecciones supra e infratentoriales con realce periférico regular con el medio de contraste. G, H: RM DWI y ADC presentando restricción central de la difusión. Hallazgos en relación con múltiples abscesos supra e infratentoriales con importante edema vasogénico perilesional.

No se ha identificado una clara relación respecto a los mecanismos inmunológicos implicados en la infección, aunque la respuesta inmune es crucial para el control de la infección. Se describe una mayor frecuencia en personas inmunocompetentes ${ }^{3}$, aun así, también se asocia a estados 
de inmunosupresión como factor de riesgo para el desarrollo de la enfermedad ${ }^{5}$. En nuestro caso no se identificó un estado aparente de inmunocompromiso.

Según su localización, el cuadro se puede clasificar en: cérvico-facial, torácica, abdomino-pélvica, musculoesquelética, del sistema nervioso central (SNC) y diseminada. En el ser humano, Actinomyces israelii es la especie más frecuentemente asociada a la enfermedad, estando presente en $70 \%$ de las infecciones oro-cérvicofaciales ${ }^{6}$. La presentación diseminada como en nuestro caso clínico, con una posible distribución hematógena, es extremadamente infrecuente, descrito principalmente en las especies A. meyeri, A. israelii y A. odontolyticus ${ }^{7}$, esta última aislada en nuestro paciente.

Durante la fase aguda de la enfermedad, se presenta un proceso inflamatorio con predominio polimorfonuclear, sin foco de infección distinguible. En la medida que el cuadro progresa, la reacción inflamatoria conduce a un cuadro de fibrosis perilesional, que se extiende a las estructuras adyacentes, sin limitación alguna ${ }^{2}$.

La localización clínica más frecuente es la cervical y orofacial, lo que guarda relación con el mayor reservorio del microorganismo. La manipulación de cualquier estructura de esta ubicación puede favorecer el desarrollo de la enfermedad y su frecuencia varía según la población estudiada entre 11 y $90 \%{ }^{8}$.

El compromiso torácico se presenta en $15-20 \%$ de los casos, el que puede ser causado por una aspiración orofaríngea, perforación esofágica o por transmisión por contigüidad de un foco cérvico-facial o abdominal y por diseminación hematógena ${ }^{3}$. Una mayor predisposición al compromiso pulmonar ha sido descrita en pacientes con alteraciones previas del parénquima pulmonar (neumonía, enfermedad pulmonar obstructiva crónica, enfisema, etc.), dada la preferencia de Actinomyces spp. de colonizar tejidos desvitalizados ${ }^{9}$.

El compromiso abdomino-pelviano representa el 20\% de los casos, generalmente secundario a una apendicitis aguda perforada. Cirugías previas, neoplasia abdominal y presencia de cuerpos extraños (en el caso de las mujeres, uso de dispositivo intrauterino por más de dos años) son otros factores de riesgo descritos. Por lo general, se evidencian como lesiones que pueden semejar neoplasias, con compromiso incluso hepático y/o pancreático ${ }^{1}$. En el caso presentado no se pudo objetivar algún foco abdominal

El compromiso del SNC, es uno de los más infrecuentes. Por lo general se produce por contigüidad de infecciones oro-cérvico-faciales o por diseminación hematógena. La afección corresponde en su mayoría a abscesos (67\%), meningitis o meningoencefalitis $(13 \%)$, actinomicoma $(7 \%)$, empiema $(6 \%)$ y absceso epidural $(6 \%)^{10,11}$. Las manifestaciones clínicas de esta localización suelen estar presentes en baja proporción, lo que da lugar a un diagnóstico tardío con un lapso aproximado de dos meses entre el inicio de la enfermedad y la consulta. El cuadro en su mayoría se presenta con cefalea y déficit neurológico focal $^{2}$. En el caso presentado, el compromiso neurológico se hizo evidente a los 30 días de su internación.

La presencia de una trombosis "inusual" de la extremidad superior izquierda hizo evaluar como primeras posibilidades una trombofilia o una neoplasia; sustentado en revisiones de aproximación a la trombosis venosa de extremidades superiores ${ }^{12}$. Los hallazgos de respuesta inflamatoria clínica y de laboratorio podían estar en relación a estos diagnósticos o ser causados por un proceso infeccioso, cuyo foco debía ser esclarecido.

El conglomerado ganglionar axilar junto con el compromiso pulmonar en la TC (engrosamiento interlobulillar lobulado y una fractura patológica de T7), inicialmente fueron sugerentes de un cuadro neoplásico. Respecto a este hallazgo, un estudio realizado por Kim y cols. en 94 pacientes reveló que en base a las imágenes pulmonares el diagnóstico inicial más común fue cáncer de pulmón $(35 \%)$, seguido de neumonía (19\%), infección por micobacterias $(17 \%)$, aspergilosis $(8 \%)$ abscesos pulmonares (5\%) y sólo en seis casos (6\%) la impresión diagnóstica inicial fue una actinomicosis pulmonar'.

El patrón radiológico de la actinomicosis pulmonar proviene de reportes de casos en los cuales se describe un compromiso predominantemente apical de distribución bilateral, con presencia de cavitación y engrosamiento pleural. Este hallazgo lo menciona en particular Dontfraid, haciendo referencia a un caso de infección diseminada por A. odontolyticus con compromiso pulmonar ${ }^{13}$, como el que fue aislado en nuestro paciente. La publicación oficial de la Sociedad Norteamericana de Radiología (RNSA) indica que la infección usualmente se manifiesta como una masa o consolidación neumónica de ubicación predominantemente en los lóbulos inferiores, que tomográficamente presenta áreas necróticas de baja atenuación con realce periférico que se acompañan de compromiso pleural (engrosamiento y derrame pleural) en más de $50 \%$ de los casos ${ }^{14}$. Excepto por la ubicación, estas características eran apreciables en el paciente presentado.

Respecto a los hallazgos imagenológicos del SNC, la descripción más común, como se mencionó previamente, es la de abscesos cerebrales; sin embargo, aunque la imagen es sugerente de un proceso infeccioso piógeno, la clínica es larvada y usualmente indolente lo que hace considerar otras alternativas, como quistes o neoplasias. Las lesiones usualmente comprometen los lóbulos frontales y temporales. En la RM se aprecia una porción central hipointensa con una pared gruesa que realza de bordes irregulares o nodulares y edema perilesional; a diferencia de los quistes o los tumores cerebrales, el centro del absceso tiene restricción a la difusión y la pared que le rodea carece de esta restricción (Figura 2) ${ }^{14}$. Las características 
mencionadas se correlacionan completamente con las imágenes de RM del caso presentado.

En cuanto al diagnóstico microbiológico, éste se confirma mediante el aislamiento de Actinomyces de un sitio estéril ${ }^{1}$; sin embargo, esto se ve frecuentemente dificultado por el uso previo de antimicrobianos o la toma y transporte inadecuado de las muestras, dado sus características de anaerobio facultativo. El cultivo es positivo en menos de $50 \%$ de los casos, la tinción de Gram de la muestra en fresco tiene mayor utilidad. El cultivo se debe procesar en medio anaerobio y su crecimiento exige medios de cultivo enriquecidos, con un tiempo de 15 a 20 días ${ }^{15}$. Por este motivo se debe comunicar al personal del laboratorio la sospecha de una actinomicosis, para tener en cuenta estas consideraciones. La identificación inicial parte de la morfología de las colonias; en los cultivos de secreción axilar y cerebral de nuestro paciente se apreciaron colonias de color naranja intenso, que característicamente corresponden a A. odontolyticus (Figura 3).

El aislamiento de $A$. odontolyticus en nuestro paciente se logró por siembra convencional en medio agar sangre en atmósfera de anaerobiosis a $36{ }^{\circ} \mathrm{C}$. Se obtuvo crecimiento a las $48 \mathrm{~h}$ de incubación obteniéndose de colonias pequeñas, secas, no hemolíticas de color salmón que con el tiempo se tornaron color naranja intenso. La morfología macroscópica de la colonia fue de aspecto grumoso de difícil dilución en solución salina. La identificación del microorganismo se obtuvo por medio del sistema automatizado Vitek 2XL Biomerieux ${ }^{\circledR}$ con tarjeta ANC que identifica microrganismos anaerobios y Corynebacterium. Además, se procesó con MALDITOF el cual fue no concluyente.

El tratamiento de elección en la actinomicosis es penicilina. En general este tipo de microorganismos son susceptibles a los $\beta$ lactámicos ${ }^{1}$. La duración recomendada es por períodos prolongados, entre seis y 12 meses, con una primera etapa de tratamiento intravenosa, de dos a seis semanas y posterior cambio a la vía oral. Incluso todo el tratamiento puede realizarse por vía oral, si las condiciones del paciente lo permite. Sin embargo, los cursos cortos o interrumpidos de antimicrobianos pueden dar lugar a una evolución tórpida con empeoramiento de los síntomas ${ }^{1-3}$. Esta situación pudo ocurrir en nuestro paciente, ya que en un inicio se optó por un tratamiento para una infección urinaria asociada, recibiendo un ciclo corto de siete días de una cefalosporina. Posteriormente, con la confirmación microbiológica se ajustó a ampicilina/sulbactam. Si bien Actinomyces spp. no produce $\beta$ lactamasas, el uso de su inhibidor permite cubrir otros microorganismos que pueden estar presentes y que como

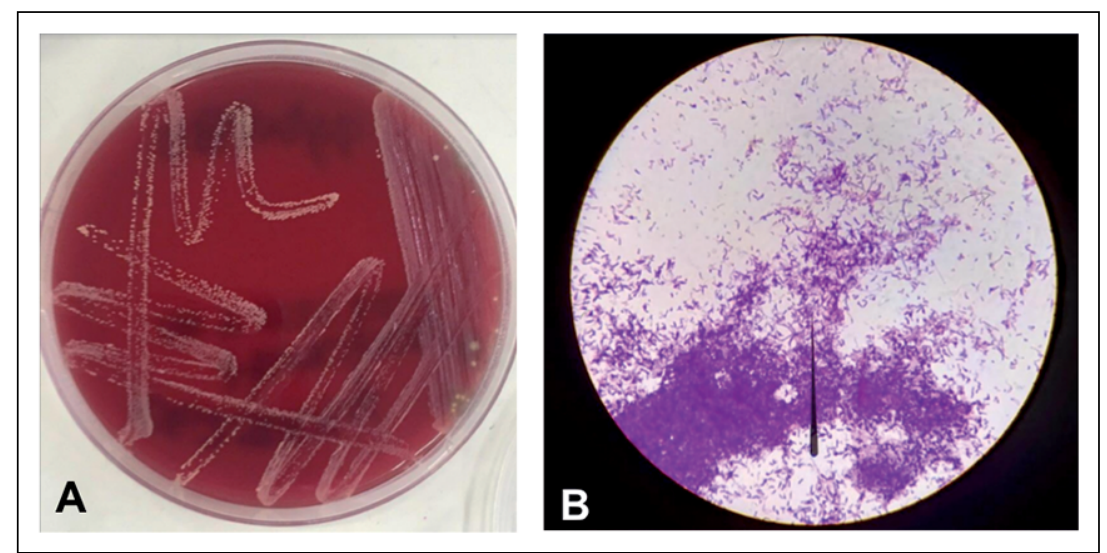

Figura 3. A: Colonia característica de Actynomyces odontolyticus. Colonias pequeñas, aspecto seco arenoso, color salmón. Crecimiento en agar sangre a $36^{\circ} \mathrm{C}$, en atmósfera anaerobia. B: Tinción de Gram de colonia de A. odontolyticus. Bacilos grampositivos cortos (100x).

se ha mencionado previamente, favorecen el crecimiento y expansión de Actinomyces spp.

El pronóstico de la actinomicosis depende del sitio de la infección. El rango de mortalidad va desde 0 a $28 \%$, siendo peor en los escenarios con compromiso del $\mathrm{SNC}^{16}$. En el caso presentado el compromiso fue extenso, por una de las especies menos comunes, en que se asoció a una enfermedad diseminada con resultado fatal.

Agradecimientos. Al Servicio de Radiología del Hospital Militar Central, en especial a Claudia Ortiz y Liliana Arias por la colaboración en la preparación y descripción de las imágenes. A Marisol Carreño quien ayudó en la elaboración y redacción del texto.

\section{Resumen}

La actinomicosis diseminada es muy infrecuente, así como la afección del sistema nervioso central (SNC) asociada, con mortalidad de hasta $28 \%$. Sus manifestaciones pueden ser similares a cuadros infecciosos de otras etiologías, por lo que el conocimiento de la entidad aumenta la sospecha clínica y permite brindar un tratamiento oportuno. Se presenta el caso clínico de un adulto con edema en una extremidad superior como manifestación de una tromboflebitis y una lesión abscedada axilar, en que se confirmó una infección por actinomicetos. Presentó una diseminación hematógena con compromiso de SNC, de evolución fatal. 


\section{Referencias bibliográficas}

1.- Valour F, Sénéchal A, Dupieux C, Karsenty J, Lustig S, Breton P, et al. Actinomycosis: etiology, clinical features, diagnosis, treatment, and management. Infect Drug Resist 2014; 7: 183-97.

2.- Herrero Martínez J A, Gómez-Gómez J, García Vázquez E, Hernández-Torres A. Actinomicosis. Medicine-Programa de Formación Médica Continuada Acreditado 2014; 11: 3419-25.

3.- Wong V K, Turmezei T D, Weston V C. Actinomycosis. BMJ 2011; 343: d6099.

4.- Schaal K P, Lee H J. Actinomycete infections in humans-a review. Gene 1992; 115 (1-2): 201-11.

5.- Manfredi R, Mazzoni A, Marinacci G, Nanetti A, Chiodo F. Progressive intractable actinomycosis in patients with AIDS. Scand J
Infect Dis 1995; 27: 405-7.

6.- Pulverer G, Schütt-Gerowitt H, Schaal K P. Human cervicofacial actinomycoses: microbiological data for 1997 cases. Clin Infect Dis 2003; 37: 490-7.

7.- Felz M W, Smith M R. Disseminated actinomycosis: multisystem mimicry in primary care. South Med J 2003; 96: 294-9.

8.- Sharkawy A A. Cervicofacial actinomycosis and mandibular osteomyelitis. Infect Dis Clin North Am 2007; 21: 543-56, viii.

9.- Kim S R, Jung L Y, Oh I J, Kim Y C, Shin K $\mathrm{C}$, Lee M K, et al. Pulmonary actinomycosis during the first decade of 21 st century: cases of 94 patients. BMC Infect Dis 2013; 13: 216.

10.- Bellesi M, Di Bella P, Provinciali L. Diagnostic difficulties with central nervous system actinomycosis. Neurol Sci 2011; 32: 945-7.

11.- Smego R A Jr. Actinomycosis of the central nervous system. Rev Infect Dis 1987; 9: 85565 .

12.- Kucher N. Clinical practice. Deep-vein thrombosis of the upper extremities. N Engl J Med 2011; 364: 861-9.

13.- Dontfraid F, Ramphal R. Bilateral pulmonary infiltrates in association with disseminated actinomycosis. Clin Infect Dis 1994; 19 : 143-5.

14.- Heo S H, Shin S S, Kim J W, Lim H S, Seon $\mathrm{H}$ J, Jung S I, et al. Imaging of actinomycosis in various organs: a comprehensive review. Radiographics 2014; 34: 19-33.

15.- Moniruddin A B M, Begum H, Nahar K. Actinomycosis : an update. Medicine Today 2010; 22: 43-47.

16.- Acevedo F, Baudrand R, Letelier L M, Gaete P. Actinomycosis: a great pretender. Case reports of unusual presentations and a review of the literature. Int J Infect Dis 2008; 12: 358-62. 